\title{
Analysis ballistic impact modeling of multicomponent fabrics with jalaba structure
}

\author{
Asmanto Subagyo \\ Department of Chemical Engineering, Concentration Textile Engineering, Faculty of Industrial Technology, Islamic University of \\ Indonesia
}

\begin{abstract}
Various textile materials have been employed in the past for protection against the ballistic with only limited satisfaction without realizing in underlying mechanism of ballistic impact. The multicomponent fabrics was prepare by nylon yarn with plain constraction with thickness of $0.18 \mathrm{~mm}$. The multicomponent fabrics were made 35 layers (laminated) with various thickness. A computational constitutive model has been developed to characterize the progressive failure behaviours of multicomponent fabrics with jalaba structure as reinforcement under high velocity impact conditions. Test result indicates that multicomponent fabric with jalaba structure can reach second level of International Standar of NIJ-010104 for hand gun (revolver) at shoot distance 5 meters. If compared with panel from biotextile composite the multicomponent fabrics have advantages in structure stability, flexibility, lightly and confortable. The integrated modeling was sucessful utilized to predict the damage and ballistic bihavior of multicomponent fabrics to various ballistic impact conditions.
\end{abstract}

\section{Introduction}

Ballistic protection is a class of protective cloting which aims at protecting the individuals from the bullets and steel fraghment from hand held weapons and exploding munitions. The use of armour to protect the personal has a long history and dates back to time immemorial. As the man developed for better clothing for protection. The list of body armour materials has included every conceivable material including the traditional material like steel and less traditional ones like silk and leather. The best known method of protecting the human body in old days from all kinds of missile was to use a hard rigid material which resisted the penetration and dissipated the load of impact. With the advent of synthetic textiles better ballistic protective systems have been developed. Although the basic idea of spreading the load over a large area is still applied, because it is better to dissipate the energy of impact by deformation and breaking the protective material (Aboot, 1999). Nylon fabric, which has high work of rupture was thought to be ideal for ballistoc protection. However, it became very clear that simple rupture is not the only creterion for ballistic protection. Due to the fact that ballistic protection process is a complex phenomenon involving the transverse velocity, tensile properties and the fine structure, however this article a brief account of multicomponent fabrics used for the desinging of ballistic protective clothing with JaLaBa structure. The analysis penetration and ballistic modeling of multicomponent fabrics has been also discussed.

\subsection{Ballistic Requirements}

The basic objective of the body armour research is to manage the conflicting goals of producing light hight yet confortable ballistic protection system with superior performance and low cost. On the other hand, for user the comfort degree mobility and maximum protection against injury are important, while for technologist, the level of protection required and energy absorption characteristics of the material are important.

According to Capilli and Rothulzen (1999), that energy absorption characteristics of the body armour are very important consist of some types namely; kinetic energy of the fabric moving towards the point of initial impact, in original fabric between the pyramid and the longtudinal wave form, strain energy accumulated in the yarns of the pyramid, energy dissipated as heat by friction between fiber and fiber or fiber and projectile.

The bullet resistant clothing has to stop bullet from penetrating and absorb its kinetic energy converting it into work deformation (Pushpa, 2009). Therefore, the primary factor which influence the performance of bulletproof material are strength and modulus and elongation at break, deformability of the projectile and the velocity of transverse shock wave in the fiber.

\footnotetext{
* Corresponding author: asmanto.subagyo@uii.ac.id
} 


\subsection{Nylon 6}

Nylon fibers were material of choice for most of the ballistic applications in early times. The advantage of nylon ussually has absorbs twice the amount of energy. The transverse wave volocity is almost 3 until 4 times higher to that in nylon. Hence stress propagation is more efficient with aramids which is clear, as showed Figure 1. The elongation of the yarn around the point the point of impact was measured approximately $9.8 \mu$ s after the shot was fire at a speed $398 \mathrm{~m} / \mathrm{s}$. It is evident that the strain nylon fiber fabric is spread over much large area and the elongation is much lower. Moreover, nylon creeps under such high strain rates at which the ballistic operate and sometimes melting and fusion at the interlacement points (Prosser R A, 1988).

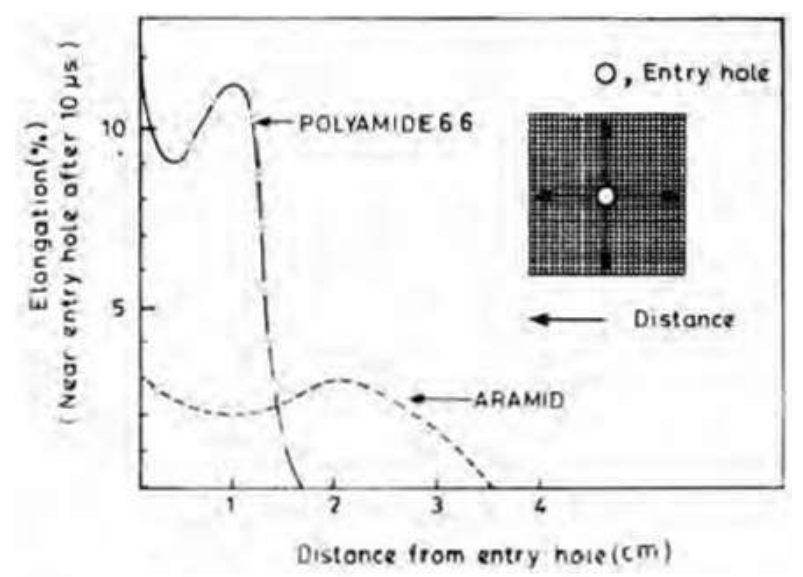

Fig 1. Comparison of ballistic between nylon and aramid fabrics

\subsection{Fabric Design for Ballistic Protection}

According to Cunniff. 1999, that to large extent the ballistic response of fabrics appears to be closely related to the ballistic response of the single yarns. In woven fabric since there is an extensive interaction among the yarns due to interlacements, it was found that tranverse deflection cause loading of the crossover yarns and up to $50 \%$ of the total energy absorption may accur in the secondary yarns. Therefore fabric construction particulars have a major role in the final ballistic protection. On the other hand, if the weave is too tight or the fabric is too stiff, deflection will be restricted, cuasing shear failure due to concentration of stress at the impact point. Therefore, it is the balance force distribution with maximum interlacements in a given unit area which makes plain weave contruction most suitable for ballistic protection.

\subsection{Desinging of Ballistic Protective Clothing}

Principally, soft body armour is constracted from multi layers of woven fabric with jalaba structure sewn together with crosswise seam. The calibre to be stopped depend on the yarn count, number of multicomponent fabric layer in making a bullet proof vest. Various fabric layer or multicomponent fabric are stacked with jalaba structure which harbours the risk that bullets impacting in succesion at not too great a distance from each other will damage the same warp or weft yarn all the multicomponent layers.

\subsection{Modeling of Multicomponent Fabrics with Jalaba Structure}

In this work, to enhance the modeling capability of the failure behaviour of multicomponent fabrics laminates due to transverse impact, a multicomponent fabrics lamina model based on the 3 Dimens stress field. The failure model can be used to effectively simulate fiber failure, jalaba stucture damage and behaviour under all conditions and sliding of failure surfaces. The major problem is that the whole process typically last only for few microseconds and it is very difficult to get specific information of all the processes that take place and then use them to validate a particular model. Furthermore, the failure modeling approach is advantageous as it enables one to predict delamination when locations of delamination sites can not be anticipated, namely locations of potential delamination initiation is caculted wuthout a prioridefinition of an inter laminar crack surface. Failure models based on the strains in a multicomponent fabrics layer with jalaba structure and improved progrssive failure modeling capability are established for a nylon plain weave fabric layer which consist of 35 layers. It can be used to effectively simulate the fiber failure and delamination behavior under high strain- rate and high pressure ballistic impact condistions. The multicomponent fabric failure criteria are expressed in term of ply level enineering strains with $\mathrm{x}, \mathrm{y}$ and $\mathrm{z}$ denoting the in plane wrap and out of plain directions, respectively. On the other hand, the associated elastic moduli are $E_{x}, E_{y}, E_{z}$ and $G_{x y}, G_{y x}, G_{z x}$. The fiber on multicomponent failure criteria for a unidirectional layer are generalized to characterize the fiber damage in term of strain components for a plain weave multilayer. The fill and warp fiber tensile damage are given by the quadratic inter-action between the associated axial and through the thickness tesile strains as follow;

$$
\begin{aligned}
& f_{1}-r_{1}^{2}=\left(\frac{E_{x}\left\langle\varepsilon_{x}\right\rangle}{S_{x T}}\right)^{2}+\left(\frac{G_{x z} \varepsilon_{x x}}{S_{x F S}}\right)^{2}-r_{1}^{2}=0 \\
& f_{2}-r_{2}^{2}=\left(\frac{E_{y}\left\langle\varepsilon_{y}\right\rangle}{S_{y T}}\right)^{2}+\left(\frac{G_{y z} \varepsilon_{y z}}{S_{y F S}}\right)^{2}-r_{2}^{2}=0
\end{aligned}
$$

Where $S_{\mathrm{xT}}$ and $\mathrm{S}_{\mathrm{yT}}$ are axial tensile strength in the fill and wrap directions and $\mathrm{S}_{\mathrm{xFS}}$ and $\mathrm{S}_{\mathrm{yFS}}$ are layer multicomponent fabric shear strengths due to fiber shear failure in the fill and wrap directions. These multicomponent failure creteria are applicable when the associated $\varepsilon_{x}$ and $\varepsilon_{y}$ is positive. In equation (1) $r_{1}$ and $r_{2}$ are the damage thresholds which are equal to one without damage.

When $\varepsilon_{\mathrm{x}}$ and $\varepsilon_{\mathrm{y}}$ are compressiive, if assumed that damage in fill and warp directions are given by the maximum strain so the equations; 


$$
\begin{aligned}
& f_{3}-r_{3}^{2}=\left[\frac{E_{x}\left\langle\varepsilon_{x}^{\prime}\right\rangle}{S_{x c}}\right]^{2}-r_{3}^{2}=0, \quad \varepsilon_{x}^{\prime}=-\varepsilon_{x}-\left\langle-\varepsilon_{z}\right) \frac{E_{z}}{E_{x}} \\
& f_{4}-r_{4}^{2}=\left[\frac{E_{y}\left\langle\dot{\varepsilon}_{y}^{\prime}\right\rangle}{S_{y c}}\right]^{2}-r_{4}^{2}=0, \quad \varepsilon_{y}^{\prime}=-\varepsilon_{y}-\left\langle-\varepsilon_{z}\right) \frac{E_{z}}{E_{y}}
\end{aligned}
$$

Note that the effect of through the thickness compressive strain on the multicomponent fabrics damage taken into account in equation (2), where $\mathrm{S}_{\mathrm{xC}}$ and $\mathrm{S}_{\mathrm{yC}}$ are the axial compressive strengths in the fill and warp directions respectively and $\mathrm{r}_{3}$ and $\mathrm{r}_{4}$ are the coressponding damage thresholds.

When a multicomponent with jalaba structure subjected to the transverse impact by a projectile, high compressive stresses will generally accour in the impact area with high shear stresses in the surrounding area between the projectile and the target material. Mean while the fiber shear punch damage due to the high shear stresses can be analysis for by equation (1) the crush damage due to the high through the thickness compressive pressure is modeled using the following criterion namely (Davies, 1995);

$$
f_{5}-r_{5}^{2}=\left(\frac{E_{z}\left\langle\varepsilon_{z}\right\rangle}{S_{F C}}\right)^{2}-r_{5}^{2}=0
$$

Where $\mathrm{S}_{\mathrm{FC}}$ is the fiber crush strengths and $\mathrm{r}_{5}$ is associated damage thresholds.

A multicomponent fabrics weaver layer can damage under shear stressing without accorance of fiber breakage, shear stresses model is given by:

$$
f_{6}-r_{6}^{2}=\left(\frac{G_{x y} \varepsilon_{x y}}{S_{x y}}\right)^{2}-r_{6}^{2}=0
$$

Where $S_{x y}$ is the layer shear strength due to shear failure and $\mathrm{r}_{6}$ is damage threshold.

Another failure analysis, which is due to the quadratic interaction between the thickness stresses is expected to be mainly a jalaba structure failure. This through the thickness multicomponent jalaba structure failure criterion is assumed to have the following form:

$$
f_{7}-r_{7}^{2}=S^{2}\left\{\left(\frac{E_{z}\left(\varepsilon_{z}\right)}{S_{z T}}\right)^{2}+\left(\frac{G_{y z} \varepsilon_{z z}}{S_{y z}+S_{S R}}\right)^{2}+\left(\frac{G_{z z} \varepsilon_{z}}{S_{z 0}+S_{S R}}\right)^{2}\right\}-r_{7}^{2}=0
$$

Where $r_{7}$ is damage threshold, and $S_{z T}$ is the through the thikcness tensile strength and $S_{\mathrm{yz} 0}$ and $\mathrm{S}_{\mathrm{zx} 0}$ are the shear strength for tensile $\varepsilon_{\mathrm{z}}$. The damage surface due to equation (5) is paralel to the multicomponent fabrics layering. When compressive $\varepsilon_{\mathrm{z}}<0$ the damage surface is considered to be closed and the damage strengths are assumed to depend on the compressive normal strain $\varepsilon_{z}$ similar to the Coulomb-Mohr theory:

$$
\mathrm{S}_{\mathrm{SR}}=\mathrm{E}_{\mathrm{z}} \tan \varphi\left\langle-\varepsilon_{\mathrm{z}}\right\rangle
$$

Where $\varphi$ is the Coulomb's friction
When damage predicted by this criterion accours within elements that are adjacent to the ply interface, the failure multicomponent is expected to be parallel to the layering multicomponent, can be referred to as delamination mode. Note that a scale factor $\mathrm{S}$ is introduced to provide better correlation of delamination area with experiments.

The scale factor $\mathrm{S}$ can be determined by fitting the analytical prediction to experimental data for the delamination area.

\section{Analysis Damage Criteria}

A set of damage variables $\omega$ with i value $=1,2, \ldots .6$ are introduced to relate the onset and growth of damage to stiffness losses in the material. The compliance jalaba structure $\mathrm{S}$ is related to the damage variables namely;

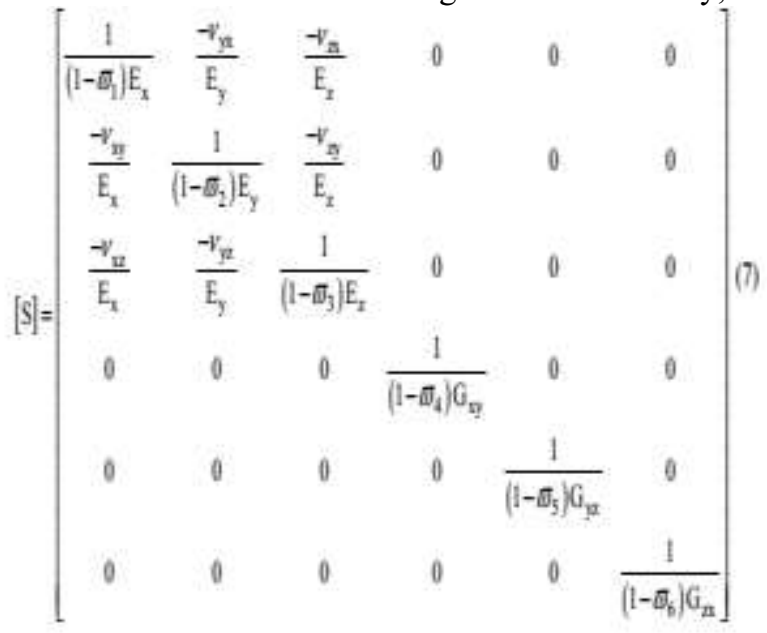

The stiffness of jalaba structure $\mathrm{C}$ is obtained by inverting the compliance jalaba stucture, $[\mathrm{C}]=[\mathrm{S}]^{-1}$.

The growth rate damage as variables $\omega_{\mathrm{i}}$ is governed by damage rule of the form

$$
\bar{\omega}_{i}=\sum_{j} \phi_{j} q_{i j}
$$

Where the scalar functions $\phi_{\mathrm{i}}$ and $\mathrm{I}=1,2, \ldots . .6$ control amount of growth and the vector-valued functions and $\mathrm{q}_{\mathrm{ij}}$ also where $I=1,2, \ldots .6$ and $j=1,2, \ldots .7$ provide the coupling between in individual damage variables (i) in the various damage $(j)$. The damage criteria $f_{i}-r_{i}^{2}=0$ of equations from 1 until 5 provide the danage surfaces in strain space. Damage growth in this case that $\phi_{\mathrm{i}}>0$, will occure when the strain path crosses the updated damage surface $f_{i}-r_{i}^{2}=0$ and the strain increment has a non-zero component in the direction of the normal to damage surface and combined with a damage growth function

$$
\sum_{j} \partial \varepsilon_{i} e_{j}=0
$$

and where $\mathrm{yi}(\mathcal{E j}, \varpi \mathrm{j})$ and $\mathrm{j}=1,2, \ldots .6, \phi_{\mathrm{i}}$ is assumed to have the form equations (9)

$$
\phi_{i}=\sum_{j} \gamma_{i} \frac{\partial f_{i}}{\partial \varepsilon_{j}} \dot{\varepsilon}_{j} \text { (nosumationover } i \text { ) }
$$




$$
\begin{gathered}
\gamma_{i}=\frac{1}{2}\left(1-\varpi_{i}\right) f_{i}^{\frac{m}{2}-1} \\
\sum_{j} \frac{\partial f_{i}}{\partial \varepsilon_{j}} \dot{\varepsilon}_{j}=\dot{f}_{i}
\end{gathered}
$$

and then for the quadratic functions of equations lead to

$$
\phi_{i}=\frac{1}{2}\left(1-\sigma_{i}\right) f_{i}^{\frac{m}{2}} f_{i} \text { (nosummationover } i \text { ) }
$$

where $\varpi_{I}$ is damage variable associated with the ith failure mode, and $\mathrm{m}$ is a material constant.

When fiber tensile damage is predicted in a layer consist of 35 layers fabrics ( one laminat) by equation (1), the load carrying capacity capacity of that layer in the associated direction is reduced to zero according to damage variable equation.

$$
\varpi_{i}=1-e^{\frac{1}{m}\left(1-r_{j}^{m}\right)}, \quad r_{j} \geq 1
$$

This provides an initial elastic region bounded by the damage functions in strain space. The nonlinear response is modeled by loading on the damage surface to cause damage growth with increasing damage thresholds and values of damage variables. After damage initiated the progressive damage model assumes linear elastic responese within the part of strain space bounded by the updated damage thresholds. Figur 2 shows typical axial tensile and compressive stress - strain curve obtained from damage model analysis under monotonic loading. Figure 2 a, shows that the model provides an initial elastic region up to strength value and softening region after damage initiated. Figure 2 b, shows stress-strain curves due to cyclic loading along the axial direction $\mathrm{x}$.

Note that the unloading and reloading stress-strain curves within the damage surface follow the reduced elastic moduls, which is given by the updated damage parameter for the axial fiber damage analysis.

For through the thicness multicomponent failure given by equation (5) the load carrying capacity within the element is assume to elastic. The load carrying behavior in through the thickness multicomponent fabrics direction is assumed to depend on the opening or closing of the jalaba structure damage. For tensile analysis when $\varepsilon_{z}>0$ the through the thickness stress multicomponents are softened and reduced to zero due to the damage criteria described above. For compressive analysis when $\varepsilon_{z}<0$ the damage surface is considered to be closed, and thus $\sigma_{z}$ is assumed to be elastic while $\tau_{\mathrm{yz}}$ and $\tau_{\mathrm{zx}}$ are allowed to reduce to a sliding friction stress.

\section{Analysis Ballistic Mechanism in Fibers}

Model analysis of ballistic impact of multicomponent were conducted by taking account for the strain-rate sensitivity properties. The analysis ballistic problem were 35 multicomponent fabric subjected to 0.5 caliber impact. The ballistic analyses were conducted using integrated with the proposed multicomponent fabric failure model.

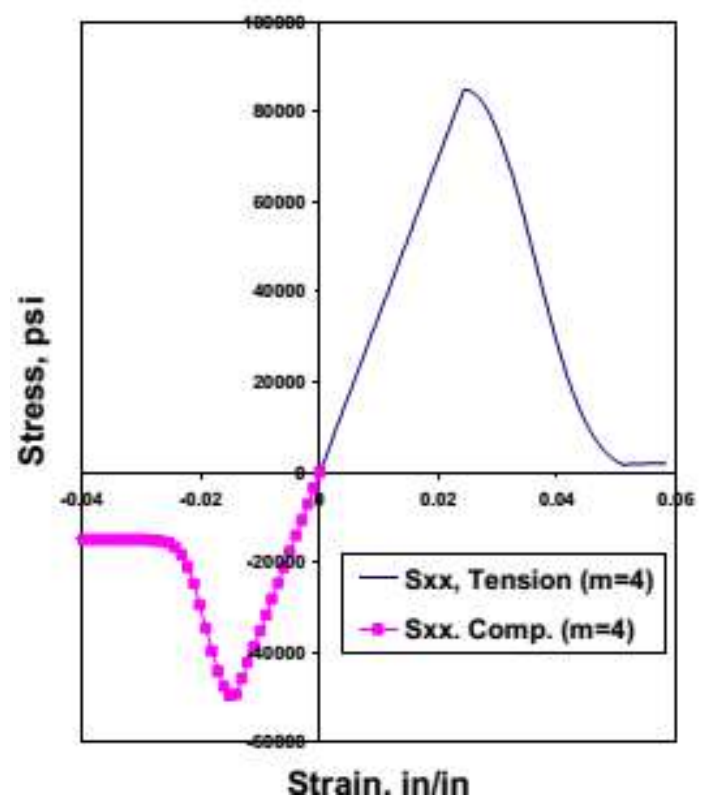

(a)

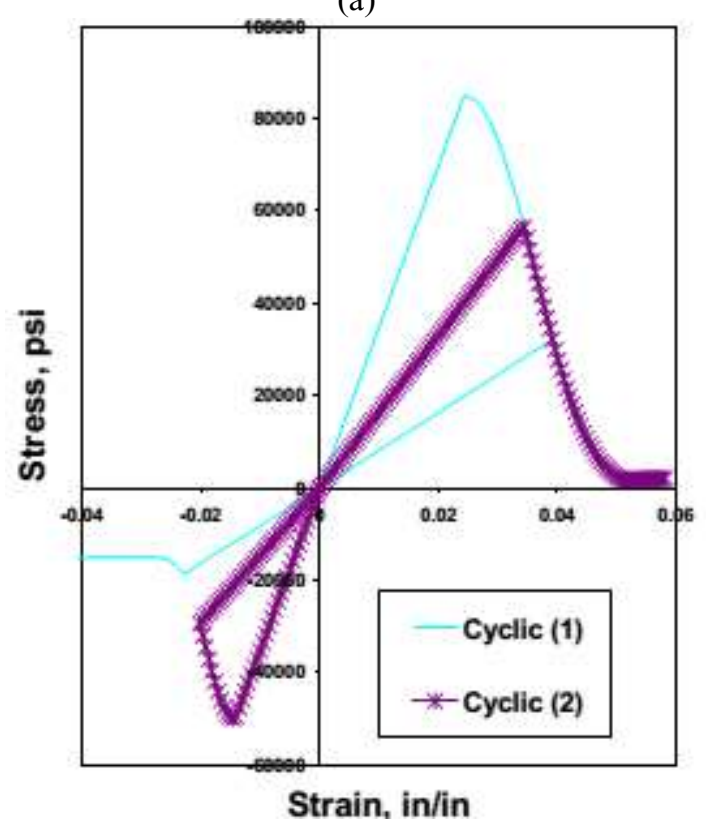

(b)

Fig 2. Stress-Strain curves for damage model under Monotonic and Cyclic loading

The finite element models for the multicomponent fabric with jalaba structure is shown in Figure 3. In these cases only one quadrant of the impact system was modeled due to the geometric and material symetry. There were 35 layers multicomponent fabrics of element through the tickness.

The basic idea of ballistic protection with fibrous materials is essentially based on the conversion of the kinetic energy into work of deformation. Hence, the principle influencing factors would be the tensile properties of the protective material, the deformability of the projectile and the armour. 


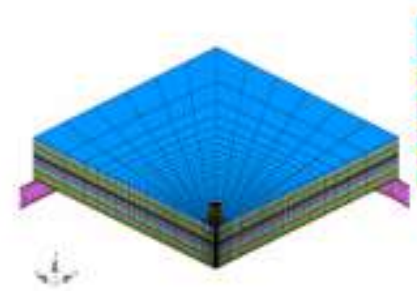

(a)

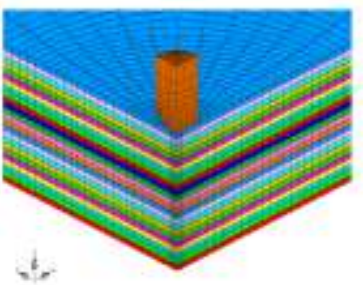

(b)

Fig 3. Finite element model for a multicomponent fabrics with jalaba structure and impactor, (a) Full view and (b) closed-up of impact area.

Figure 4. the mechanism of deformation when single yarn is subjected to impact by a projectile, with the longitudinal axis of the fiber perpendicular to the path of projectile. The impact leads to longitudinal wave propagation velocity $\mathrm{C}$ is nothing but speed of the sound in the material which can be expressed in term of density and modulus as under: $\mathrm{C}=(\mathrm{E} / \rho)^{1 / 2}$ where $\mathrm{C}$ is speed of longitudinal wave velocity, $\mathrm{E}=$ initial moduls and $\rho=$ density.

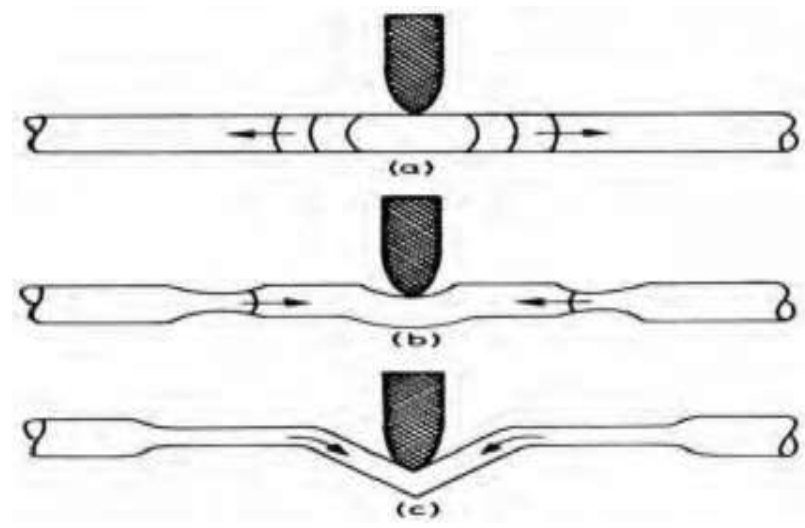

Fig 4. Failure mechanism of single fiber under ballistic impact. (a) Propagation of langitudinal compressive waves (b) Reflection as tensile wave (c) Elongation of fiber

The wave reaches the end of the fiber and is reflected back as a tensile wave. The reflection of the tensle wave is necessary so as to satisfy the boundary condition of zero stress at the fiber ends. The moment of tensile wave back towards the point of impact causes the flow of material in the same direction. Ultimately, a tensile strain is experienced by the fiber in contact with the the projectile and the magnitude of which is dictated by the impact velocity of the projectile. Continued wave reflections intensify the tensile strain with energy absorbed by the fiber being proportional to this strain (Subagyo. A. 2014). At the same time, a second and slower wave propagates along the transverse axis and cross the diameter of the fiber, moving paralel to the projectile and at the same velocity. Because of this transverse wave, the fiber starts moving in the same direction as the projectile path. The fiber continues to absorb energy and deflects until projectile is decelerated and stopped or another words the fiber strains past the dynamic yield point and breaks.

\section{Conclusions}

To predict the performance of analysis ballistics impact on multicomponent fabric used jalaba structure has succeeded in the accounting for all complexities that are involved with the penetration mechanism of the ballistic protection. The major problem is that the whole process typically last only for few microseconds and it very difficult to get the specific information of all processes that take place and then use them to validate a particular model.

This analysis model multicomponent fabric used jalaba structure used a technique which requires complex experimental to augment the numerical calculations. The success of this model is impeded maily due to the simplification of assumptions that are made ans axcessive dependence on the experimental data.

The model took care of the essential physics of impact mechanism along with impact mechanics, still keeping the solution reasonably tractable. The main assumption is that a multicomponent fabric can be thought as a single laminated and the impact is approximated as the point impact. The model successfully indicated that the majority of the energy absorbed by the fabric under ballistic impact was due to the strain and the kinetic energy in the yarns directly in contact with the projectile.

The authors gratefully acknowledge the financial support of the specialized Research Fund for the Directorate of Research and Community Service, Islamic University of Indonesia Yogyakarta.

\section{References}

1 Abott T.A., Protective Clothing, Shirley Publication, Manchester. UK (1999).

2 Davies, G A. Impact Damage Prediction in Carbon Composite Structure, International Conference on Material. Washington, USA (1995)

3 Capilli $\mathrm{K}$ and Rothulzen., High Performance Textile, Textile Research Journal, Prince, South Carolina, USA (1992)

4 Cunniff. P.M, Characteristic of Body Armour, Textile Research Journal, Prince, USA (1999)

5 Pushpa Bajaj, Ballistic Protective Cloting, Indian Journal of Fiber and Research. New Delhi, India (2009)

6 Subagyo. A. Model Impact Resistance of Bio-Textile Fabrics Composite from Agave and Pineapple Leaf Fibers. Full Paper Proceeding ETAR-2014, Vol. 1, 320-328 ISBN: 978-969-9948-23-7 . Filipine (2014) 